\title{
MIX INDUCED BY SINGLE SHOCK PASSAGE THROUGH A MATERIAL INTERFACE
}

\author{
V.C. Rupert \\ W.P. Crowley \\ G.D. Kramer
}

This paper was prepared

for submittal to the

Proceedings of the 19k International Symposium on Shock Waves

Marseille, France

July 26-30, 1993.

July 1993

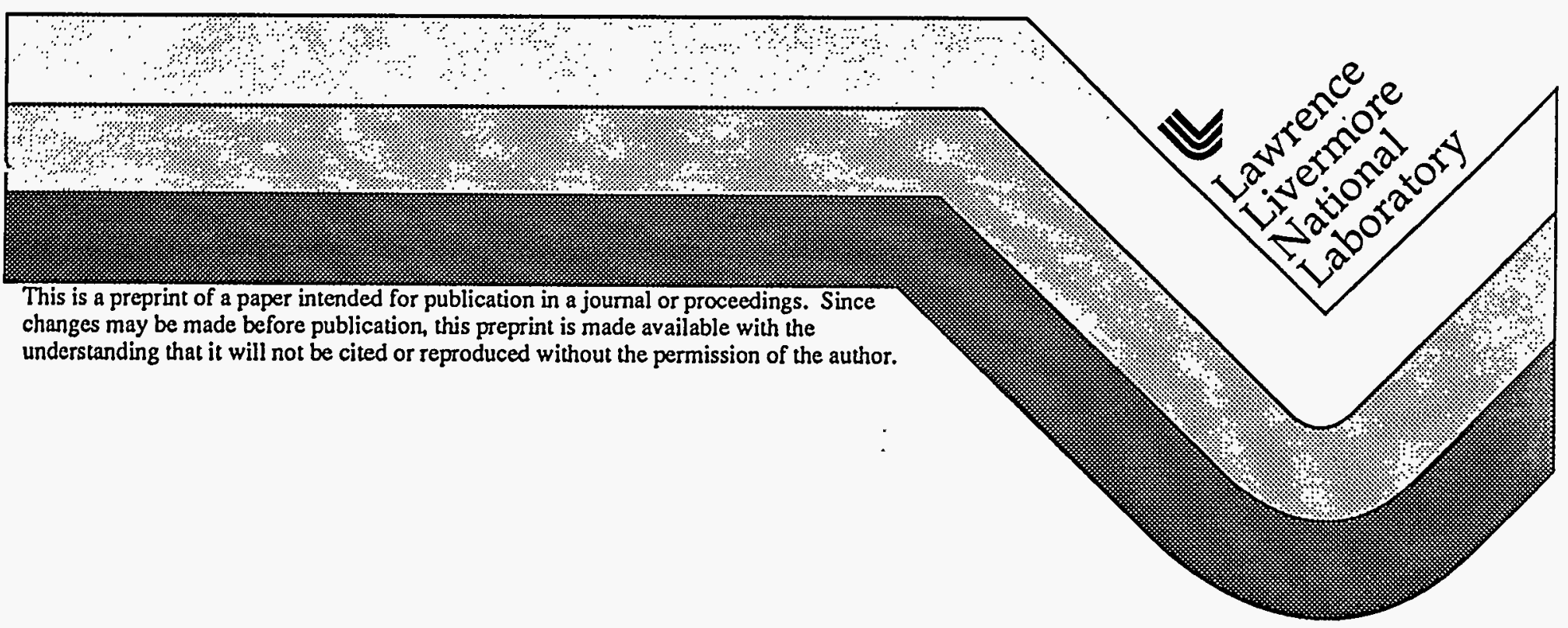




\section{DISCLAIMER}

This report was prepared as an account of work sponsored by an agency of the United States Government. Neither the United States Government nor any agency thereof, nor any of their employees, make any warranty, express or implied, or assumes any legal liability or responsibility for the accuracy, completeness, or usefulness of any information, apparatus, product, or process disclosed, or represents that its use would not infringe privately owned rights. Reference herein to any specific commercial product, process, or service by trade name, trademark, manufacturer, or otherwise does not necessarily constitute or imply its endorsement, recommendation, or favoring by the United States Government or any agency thereof. The views and opinions of authors expressed herein do not necessarily state or reflect those of the United States Government or any agency thereof. 


\section{DISCLAIMER}

\section{Portions of this document may be illegible in electronic image products. Images are produced from the best available original document.}




\title{
MIX INDUCED BY SINGLE SHOCK PASSAGE THROUGH A MATERIAL INTERFACE
}

\author{
VC Rupert, WP Crowley, and GD Kramer \\ Lawrence Livermore National Laboratory, Livermore, CA, USA
}

\section{Introduction}

The question of perturbation growth at a shocked interface between materials with different densities has been studied in earnest in the last few years (Rupert 1991). We have been interested in the case of a single-shock crossing the interface as a counterpart to the work done with one or several reflected waves (Rupert 1991). Using the LAM code (Kramer et al. 1993) we are systematically studying the interaction of a single shock with a wavy interface. During this first phase, we concentrated on the effect of the initial interface shape and on the Mach number dependence of the mixing process. Our simulations addressed a shock incident from a high-density gas onto a low-density gas interface, and from a low-density gas onto a highdensity gas interface.

\section{The numerical "experiment"}

The starting point selected for our computations was one of the cases that was used for simulation comparisons at the Third Intemational Conference on the Physics of Turbulent Mixing (ICPTM); it was based on experiments run by Zaitsev (Rupert 1991). Kramer (1993) has described the initial conditions for the simulations discussed herein.

Figure 1 shows the interface shape for case 8 , which comprises sixteen wave numbers and has a 30\% maximum amplitude-to-wavelength ratio. This case was one of those used to investigate more intense mode coupling.

\section{The effect of initial conditions}

The principal numerical diagnostic used was suggested by the experimental data and has been the mainstay of this type of work; it is the "mix width." This width is characteristic of the visual impression, which one gets from of an image of a perturbed region, obtained by integrating imaging techniques, such as schlieren and $\mathrm{x}$-ray radiography. It is characteristic of the extent of large-scale features in the flow. As pointed out by Bonazza (1991), the mix width varies depending on the imaging technique used; the calculated quantity is closer to the quantity derived from $x$-ray radiography because it directly relates to the density of the denser element.

Figure 2 shows density contours for one calculation. The large-scale features present two different aspects. One is the narrow fingers of heavy material, called spikes, that penetrate the lighter material. The other is the wide regions of light material, called bubbles, that penetrate the heavier material. In Fig. 2, the spikes have been slowed down by drag and are strongly influenced by the vorticity generated by the non-collinear density and pressure gradients that occurred when the shock crossed the original perturbed interface. As the tips of the spikes curl, the coupling between modes intensifies. The tips of the spikes can break off, as seen in Fig. 2 , leaving "chunks" of heavy material in the lighter one.

Figure 3 summarizes our results for the Ar $>\mathrm{He}$ case. The growth of the perturbations, just after compression due to shock transit, follows the expected pattem. At very early time the growth is linear and the growth rate roughly correlates with the maximum value of the

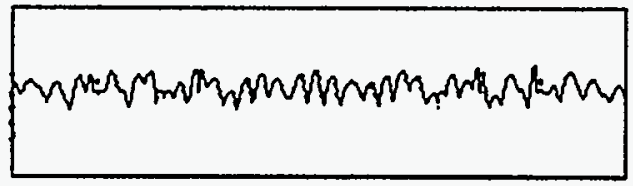

Fig. 1. The initial perturbation for case 8 , using sixteen wavelengths. Not to scale; horizontal scale is $60 \%$ of vertical. 


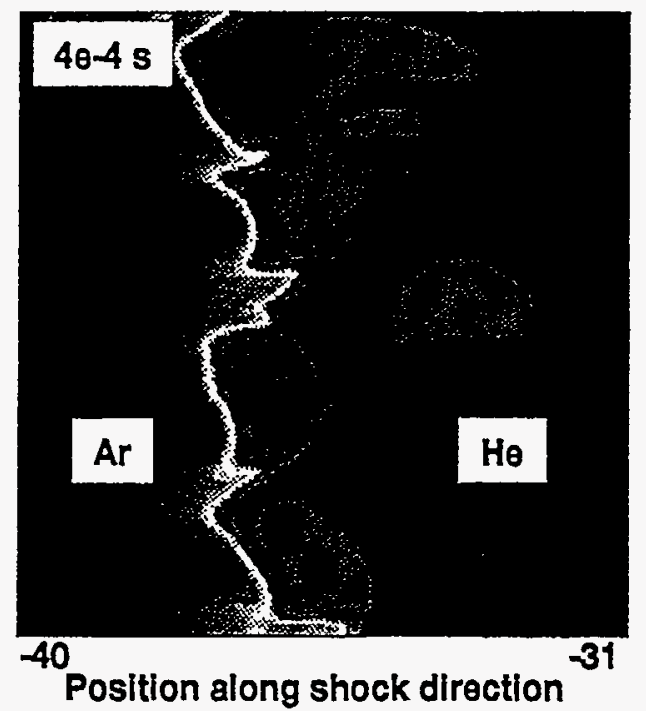

Fig. 2. Typical example of the density distribution showing spikes to the right and bubbles to the left of the perturbed region $(\mathrm{He}>\mathrm{Ar})$.

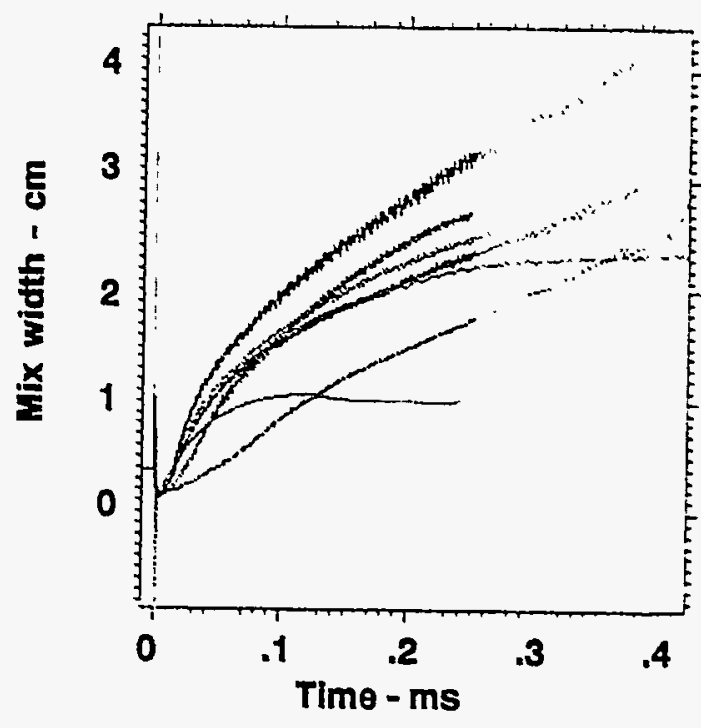

Fig. 3. The evolution of mix width over time for the $\mathrm{Ar}>\mathrm{He}$ calculations. Case 8 is represented by the purple curve.

amplitude-to-wavelength ratio (a/ $/$ ). Saturation then sets in; it occurs earlier for larger a/ $/ 2$ 's as is dramatically evidenced by the curve corresponding to the initial interface shown in Fig. 1.

It is interesting to note that the symmetry of the single-wavelength perturbation (green curve) contributes to maintaining a quasi linear growth for a longer time than would be expected based on the behavior of the multi-wavelength cases. Indeed in this situation, mode coupling can only occur to harmonics and sub-harmonics of the initial wavelength.

The effect of mode coupling (Rupert 1991) is displayed in Fig. 4, which shows density contours for two perturbations with the same distribution of wavelengths and the same amplitude variance. The initial amplitude distribution shown in case "b" leads to more efficient mode coupling. Figure 4 also explains why the mix width can actually decrease in time. The vorticity near the tips of the spikes causes them to bend toward neighboring spikes thereby decreasing the average width over which large density gradients occur. Such a decrease in mix width is not associated with demixing, which has been postulated in the case of purely Rayleigh-Taylor unstable flows (Rupert 1991). Closer inspection shows that a small amount of heavy material remains at the maximum tip position and continues to expand. However, its density is small enough to result in a long thin tail on the average volume-fraction distribution, which is not included in the range selected to define the mix width. By contrast, if an "end wall" is positioned in the shock tube, the reflected wave interacts with the interface, the pattems become much more irregular, and the growth rate increases dramatically. This has been demonstrated in earlier experiments and simulations (Rupert 1991).

While the mix width characteristic of the region of flow over which large-scale mix occurs is a tangible quantity that is easily identified in an experiment, its value is limited. A more meaningful quantity is the "atomic mix" that occurs between two materials in close contact. For example, atomic mix allows chemical reactions to take place. This component of mix, shown in Fig. 2, is the thin region that lies along the boundary of the spikes and bubbles and is were density changes from that of pure upstream to pure downstream material. We have defined a rough measure of the characteristic length of the atomic mix, called equivalent mix width (EQMW). This equivalent mix width is obtained by adding the volumes of all the zones 

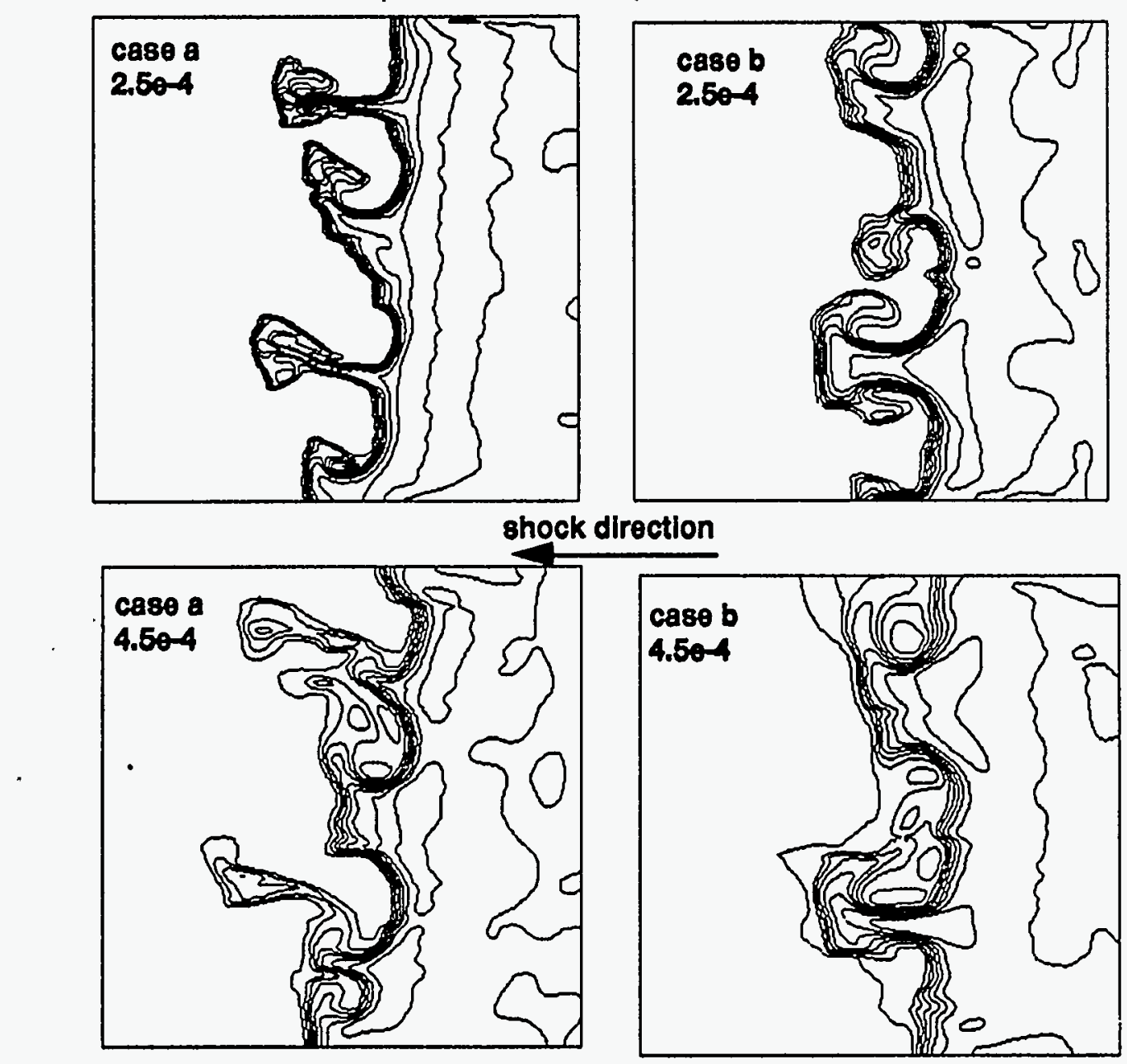

Fig. 4. Density contours for two calculations. Both started with the same wavelength distribution, amplitude variance, and incident Mach number. Two different times are shown to illustrate the effect of mode coupling.

where the volume fraction of either component is neither 0 nor 1 , and by dividing it by the shock-tube width. Thus, it is the width of a region spanning the shock tube with a volume that is equal to the volume of fluid for which the scale of mix is less than a zone width (in our case $250 \mu \mathrm{m}$ ). Better characteristics of atomic mix, such as the mass of the mixed materials and the "flame length," or length of the boundaries between materials along the spikes, bubbles, and detached chunks, are being added to the LAM code.

After a period that is much greater than the period over which these calculations were run (even though the interface has moved more than 400 times the initial perturbation width), the mode coupling should eventually result in small-scale mixing between the components. However, as in the Kelvin-Helmholtz experiments, a small amount of small-scale mixing evolves along the boundaries of the large-scale features even at early time. This mixing is initiated by the high-frequency perturbation associated with the intersection of the mesh with the nominal interface. It therefore can be expected to behave much like the high-frequency perturbation shown in Fig. 1 with its rapid saturation at a fixed level. Note that these perturbations are the computational equivalent of machining irregularities. While errors as large as 50\%, due to numerical diffusion, are associated with this value, particularly at early times, the growth of the EQMW is of the order of that of the mix width for the time of the simulations. The 
atomically mixed component appears to rapidly reach 30 to $50 \%$ of the overall mixed-region width for most of the perturbations studied. We are anticipating that up coming expansions of the code will include the capability to obtain atomically mix masses and to pursue the calculations to later times, which will allow us to study the atomic mix in greater detail. In particular, it will be quite interesting to determine whether the same correlation between large-scale and atomic mix still holds in the 3-D simulations.

The code's recent capability to extract time-dependent EQMW from the computations is too new for a full set of data to be available, and the computations have not been pursued long enough for mode coupling to completely destroy the memory of the initial perturbation. Hence, we cannot rule out the existence of an asymptotic solution that is dependent only on the amplitude variance at very late time. However, these calculations have been run long enough for practical applications. Alas, the conclusion we presented in our original abstract, which was based on results for early-time growth, does not hold true. We have to accept the fact that for a moderate number of wavelengths, no asymptotic solution is reached, and therefore, models based on amplitude variance alone cannot adequately describe the mixing process.

\section{The Mach number effect}

One of the perturbed interface shapes used in the preceding series (perturbation 2, see Kramer et al. 1993) was selected as the basis for the Mach number study in the case of Ar $>$ He.

We anticipated that shock induced radiation and electron conduction would be incorporated into LAM by the $19^{\text {th }}$ ISSW, but these physical phenomena have not yet been added. Differential preheat of the gases across the interface by radiation from the hot argon behind the incident shock (Zeldovich et al. 1991) would modify the Atwood number. Pressure equilibrium across the interface would also be perturbed, resulting in motion prior to shock arrival and further change in the relative densities. For the highest Mach number used in these calculations, $M=50$, the temperature behind the incident shock is of the order of $2.10^{5} \mathrm{~K}$. These effects are not expected to be very significant.

To facilitate comparison of the results, the data are plotted versus interface position, by analogy with Richtmyer's linear theory (Rupert 1991). Figure 5 shows the evolution of the mix width for Mach numbers ranging from 1.32 to 50 (Rupert 1991). At early time (small displacement) the growth rates scale roughly like the interface velocity except for the lowest Mach number, this is consistent with linear theory. According to Richtmyer, the perturbation growth rate is also proportional to the initial amplitude ("initial" meaning, just after shock transit through the interface). An estimate of the compression factor shows that the initial growth rate of the Mach 1.32 perturbation should be larger than for all other cases, while for Mach 3.45 it might be slightly smaller. Note that the significant increase in the growth rate for the Mach 1.32 case, $\sim 8 \mathrm{~cm}$, is due to the expansion wave reflected off the upstream boundary. Indeed, for this low Mach number, the expansion wave reflected off the interface does move upstream from the original interface position.

Figure 5 shows that the higher the Mach number, the faster the mix width reaches saturation, and the lower the saturation level. For sufficiently high Mach numbers, the asymptotic values are indistinguishable. This behavior results from the non-linear forces (whether one chooses to think about drag or vorticity), which act on the developing spikes to retard their growth. As an example, for a displacement of $\sim 9 \mathrm{~cm}$ (see Fig 5) the maximum vorticity is a factor of 10 larger at Mach 50 than at Mach 3.45, while the velocity is a factor of 16 larger. Recall that the data are plotted with a time scaled by velocity and that drag is proportional to velocity squared. Also note that the flow is subsonic behind the transmitted shock only for the incident Mach number of 1.32 .

Hence, these calculations show that, as in shear flows, high Mach numbers ( 9 or more for the case considered here) result in minimum mix for interface displacements that are more 


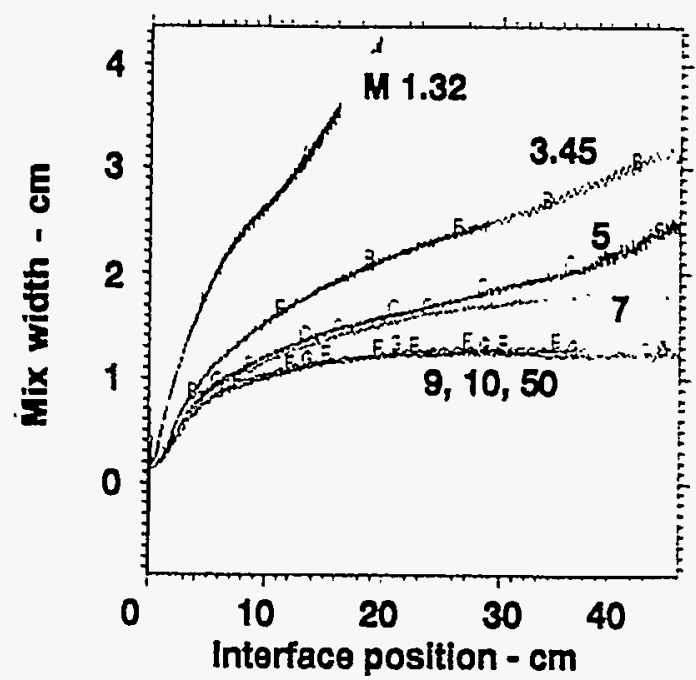

Fig. 5. Evolution of the mix width for various Mach numbers.

than a few centimeters. Even though growth rates increase with the Mach number in the linear phase of the perturbation growth, the more rapid saturation severely limits the amount of mix that can be obtained.

\section{Energy coupling}

Another flow characteristic used extensively is the fluctuating kinetic energy transferred from the incident shock to the perturbed flow. This energy is embodied in the (Favre averaged) Reynolds stress $\left(R_{x x}=\left\langle\rho u^{2}\right\rangle-\langle\rho u\rangle^{2} /<\rho>\right.$, and like terms). It is identically zero until the (planar) shock reaches the interface where the transmitted shock and reflected wave are perturbed resulting in a non-uniform density and velocity distribution in their vicinity as well as at the interface proper. Soon after the incident shock completes its transit of the interface (Besnard et al. 1989), the coupling reaches a maximum and the characteristic energy transferred from the incident to the perturbed component of the flow is obtained at that time. This is the quantity used for the initial condition in many turbulent models. We have used the nomenclature FKE to represent $R_{x x}+R_{y y}$.

Table I summarizes the results from our simulations. In general, these results are within a factor of 2 of the values obtained from Leith's (1985) linear analysis:

$$
F K E=\pi a^{2} / 2 \lambda V^{2} A t \#^{2}\left(\rho_{2}+\rho_{1}\right) \approx 0.5 \int\left(R_{x x}+R_{y y}\right) d x,
$$

where $V$ is the interface velocity, At\# $=\left(\rho_{2}-\rho_{1}\right) /\left(\rho_{2}+\rho_{1}\right)$, and $\rho_{1}, \rho_{2}$ are the densities on either side of the interface. One of the difficulties in applying this formula is the choice of the amplitude and Atwood number (Rupert 1991). In the linear approximation Leith used, the ratio $\mathrm{a}^{2} \lambda$ is replaced by a sum of such terms for the multiple wavelength perturbations. Because of the differences in amplitude distribution among the wavelengths, the $\Sigma\left(a^{2} / \lambda\right)$ differs somewhat between the cases studied for a single Mach number. A systematic trend relating variations in computed FKE and $\Sigma\left(a^{2} \lambda\right)$ is not apparent. Since in the cases studied here the individual values of $\mathrm{a}^{2} \lambda$ ranged from 0.001 to 0.074 for all perturbations, except case 8 , the linear analysis may

Table I. Energy transferred from the shock to the perturbed flow for various amplitude distributions.

$\begin{array}{lccccccc}\text { Case } & \mathbf{2} & \mathbf{3} & \mathbf{4} & \mathbf{5} & \mathbf{6} & \mathbf{7} & \mathbf{8} \\ \Sigma\left(\mathrm{a}^{2} / \lambda\right)(\mathbf{c m}) & 1.5 \mathrm{e}-2 & 1.5 \mathrm{e}-2 & 1.5 \mathrm{e}-2 & 2.1 \mathrm{e}-2 & 4.7 \mathrm{e}-3 & 8.1 \mathrm{e}-3 & 1.3 \mathrm{e}-1 \\ \mathbf{R}_{\mathbf{X x}}\left(\mathrm{ergs} / \mathbf{c m}^{2}\right) & 1.90 \mathrm{e} 5 & 1.96 \mathrm{e} 5 & 1.93 \mathrm{e} 5 & 1.81 \mathrm{e} 5 & 2.01 \mathrm{e} 5 & 1.96 \mathrm{e} 5 & 8.23 \mathrm{e} 4 \\ \text { FKE }\left(\mathrm{ergs} / \mathbf{c m}^{2}\right) & 2.07 \mathrm{e} 5 & 2.06 \mathrm{e} 5 & 2.08 \mathrm{e} 5 & 1.97 \mathrm{e} 5 & 2.20 \mathrm{e} 5 & 2.00 \mathrm{e} 5 & 1.06 \mathrm{e} 5\end{array}$


still have been applicable. For case 8, however, the range was 0.006 to 0.29 ; the linear analysisclearly does not apply. In this case, the calculated FKE is a factor of 2 lower than for other perturbations with the same amplitude variance. Results of the numerical simulations indicate that in the non-linear regime, which is representative of real interfaces, the efficiency of energy transfer between the incident shock and the perturbed flow field decreases as the maximum ratio of amplitude-to-wavelength increases, which is contrary to the linear theory results. This effect warrants closer scrutiny.

\section{Conclusion}

A systematic study of the coupling of a single shock with a perturbed interface has been initiated. While this study is far from complete, a number of interesting facts have emerged. Results show that for a moderate number of wavelengths, even if the amplitude-to-wavelength ratios are less than $10 \%$, no asymptotic solution is reached. This indicates that models based on amplitude variance alone cannot adequately describe the mixing process. Our simulations show that this also applies for the saturation level obtained with high amplitude-to-wavelength ratios (see Fig. 1). More information concerning the spectrum of the interface shape must be used in models wishing to represent the evolution of a perturbed interface from its initial shape to its fully turbulent state. Mode coupling plays a significant role in limiting the growth rate of the mixed-zone width so that for multiple wavelengths with large amplitude-to-wavelength ratios the growth rate saturates rapidly. More work is needed to determine the systematics of this case.

The evolution of mix width is one measure of the mixing process. We are exploring other measures, such as the amount of atomically mixed material and the distribution of chunk sizes. These parameters are more relevant to the physical phenomena resulting from mix and are required by newer models of the mixing process.

We have also confirmed that in spite of the initially higher growth rates associated with higher Mach numbers, the increased drag dominates the perturbation growth, and the mix is limited as Mach number increases. Hence, modelization of drag can be tested against the calculations presented here. Mach number does not seem to have any other special effect on the evolution of the perturbation.

Finally, our calculations imply that the functional relationship between flow variables and energy transfer to the perturbed field obtained from the linear theory is good to a factor of 2 (Besnard et al. 1989). However, for a highly non-linear interaction, the energy coupling appears to decrease.

\section{References}

Rupert V (1991) "Shock-Interface Interaction: Current Research on the Richtmyer-Meshkov Problem," Proceedings of the $18^{\text {th }}$ ISSW, Sendai, Japan.

Bonazza R (1991) CIT Doctoral Thesis (or R. Bonazza, B. Sturtevant Measurements by X-Ray Densitometry of Shock-Excited Turbulent Mixing at an Air-Xenon Interface).

Kramer GD, Crowley WP, and Rupert VC (1993) "Locally Adaptive Remeshing Scheme for Calculating Fluid Instabilities," Proceedings of the $19^{\text {th }}$ International Symposium on Shock Waves, Marseille, France.

Zeldovich YB and Raiser YP (1967) Physics of Shock Waves and High Temperature Hydrodynamic Phenomena, Academic Press.

Besnard DC, Haas JF, and Rauenzhan RM (1989) "Statistical Modeling of Shock-Interface Interaction," Physica D, 37, 227-247.

Leith CE (1985) private communication, Lawrence Livermore National Laboratory, Livermore, CA, USA.

Work performed under the auspices of the U.S. Department of Energy by the Lawrence Livermore National Laboratory under Contract W-7405-Eng-48. 